\title{
RINGO: A DEMONSTRATOR OF WDM OPTICAL PACKET NETWORK ON A RING TOPOLOGY
}

\author{
Andrea Carena, Valter Ferrero, Roberto Gaudino, Vito De Feo, Fabio Neri, \\ Pierluigi Poggiolini \\ Dipartimento di Elettronica - Politecnico di Torino \\ Corso Duca degli Abruzzi, 24 - 10129 Torino - Italy \\ optcom @polito.it
}

\begin{abstract}
This paper presents the work carried out by the Optical Communication Group at Politecnico di Torino in the framework of the RINGO project. The RINGO network is based on a WDM optical ring, with an input queuing access protocol and multicast capabilities. In this paper we present the network architecture and node structure, together with the results obtained on the RINGO experimental demonstrator. We investigate several important transmission issues, such as EDFA gain locking, and the implementation of several subsystems, such as node controller, $\lambda$-monitor and packet transmitter.
\end{abstract}

\section{Introduction}

Wavelength division multiplexing (WDM) is a well-established technique to exploit the fiber bandwidth in both transport and access networks, and all major vendors in this field offer a wide range of products and commercial solutions. At the research and standardization levels, the focus is now shifting from the use of optical technologies for implementing transmission functions to the development of an "Optical Layer" capable of implementing network functions such as switching, protection and restoration in the photonic domain [1], possibly under electronic control. The International Telecommunication Union (ITU) and other international committies are working towards the standardization of protocols and control techniques for wavelength routed optical networks [2], i.e., systems in which the optical layer is used not only as a "raw" point-to-point high bit-rate data pipe, but also to implement some network reconfiguration capabilities, thus introducing traffic adaptation, restoration and protection. $R e-$ configurable optical networks (or Automatically Switched Transport Networks - ASTN) started to be studied by consortia such as MONET [3], and are now a 
hot issue for the manufacturers of optical systems. These solutions are aimed to developing a flexible network that can be reconfigured on time scales in the order of milliseconds or greater to react to system failures or traffic changes.

On the contrary, the implementation of optical packet switching functions (i.e., of an optical layer that can handle data traffic on time scales in the order of microseconds or less) [4] is still at an earlier development phase, although several testbeds have already been proposed [5],[6],[7]. This is certainly due to the greater technological challenges that have to be solved when dealing with packets directly at the optical level, but also to the fact that current networks were optimized for the existing electronic technologies. Indeed, although optical devices allow huge potential in terms of available bandwidth, they do not easily offer several features in terms of handling and storage of digital signals, which are very natural and easy in the electronic domain.

In this paper, we present network architecture, resource allocation protocol, design, and experimental results of the RINGO (RING Optical network) project, a consortium of Italian Universities coordinated by the Optical Communication Group at Politecnico di Torino, Italy. The RINGO project is focused on experimentally studying the feasibility of a WDM optical packet network based on a ring topology. The RINGO network envisions the use of slotted TDM/WDM optical rings for the implementation of packet switched MAN and WAN networks. Currently, high capacity MAN/WANs are implemented using the consolidated framing and protection techniques of SONET/SDH WDM rings, where SONET/SDH frames are used as "containers" for (variable-size) IP packets or (fixed-size) ATM cells. This approach, although highly consolidated from a technological point of view, does not offer a great flexibility and is not optimized for switching packetized data. Moreover, each node of a SONET/SDH WDM ring must optoelectronically convert the aggregate bit rate on each input link, handle it electronically and re-convert it to the output transmitters. This approach does not scale well: with the current increase of transmission requirements, we easily end up with nodes based on electronic routers that should handle aggregate bandwidths in the order of several Tbit/s.

The experimental part of the RINGO project required the implementation of the basic building blocks of a node, focussing also on:

- hardware demonstration of the MAC protocol, which includes advanced features such as multicasting and efficient queueing algorithms, and a simple but effective channel inspection (or $\lambda$-monitor) technique;

- detailed investigation on transmission issues such as power budget, EDFA gain locking and optical filtering.

The paper is organized as follows. The RINGO general architecture and the MAC protocol are explained in Sect. 2, together with a review of the main features of the proposed network. The following Sect. 3 describes the current RINGO experimental setup, consisting of two node-prototypes that can handle 
four channels at $2.5 \mathrm{Gbit} / \mathrm{s}$ with $1 \mu \mathrm{s}$ packet duration. In the same section, a detailed description of the hardware implemented for the node controller is given. Sect. 4 addresses, both experimentally and theoretically, several fundamental issues related to the design of the RINGO network at the optical transmission level, such as power budget constraints and EDFA gain locking. Comment and conclusions are then drawn in Sect. 5.

\section{RINGO Architecture and Protocol}

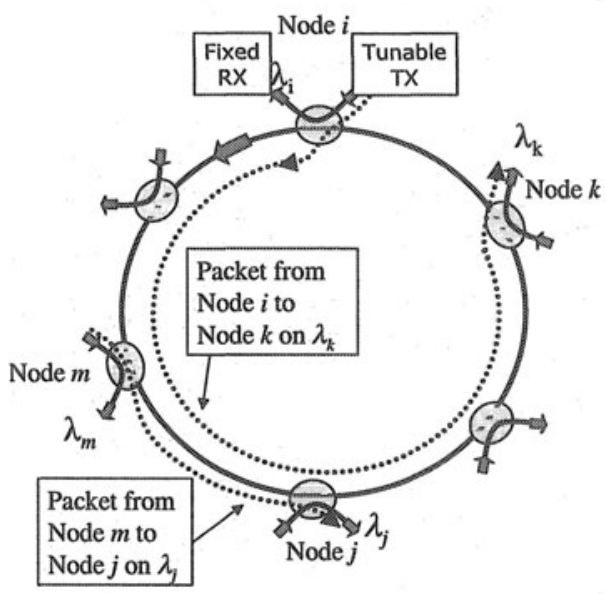

Figure 1. Architecture of the RINGO network.

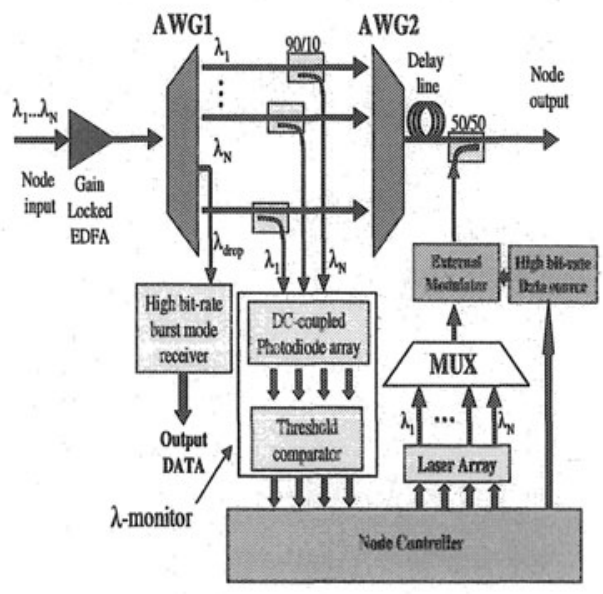

Figure 2. Structure of a RINGO node.

The general architecture of the RINGO network is shown in Fig. 1, while the structure of a node is shown in Fig. 2. RINGO is based on a unidirectional fiber ring with $N$ nodes implementing the interface between electronic and optical domains. Each node queues at the electronic level fixed-size packets to be sent on the ring. The main features of the architecture are:

- the number $N$ of nodes in the network is equal to the number $N$ of used wavelengths (which will be often indicated in the following as "channels"); extensions to number of nodes larger than the number of wavelengths are possible [10], but are not considered here;

- each node optical interface is equipped with a tunable transmitter, and with a fixed receiver operating on a given wavelength $\lambda_{i}$ that identifies the node itself; in order to communicate to node $k$, a node must tune its transmitter to send a packet on $\lambda_{k}$, as shown in Fig. 1. Furthermore, each node must remove the information carried by its wavelength $\lambda_{i}$ from the ring. If required, some particular nodes may receive more than one wavelength, by simply dropping them and replicating the receiver subsystem. 
- each node is able to check the status of the wavelength occupation (a feature called $\lambda$-monitoring in the rest of the paper) on a slot-by-slot basis, and is thus able to avoid collisions by a generalization of the empty-slot approach, which allows to transmit packets only in those slots that are actually available;

- the packets to be transmitted are queued using a Virtual Output Queueing (VOQ) [8] algorithm, i.e., by storing packets waiting for ring access in separated queues, each corresponding to a different destination or to a different set of destinations. It is worth noting that VOQ was demonstrated to achieve $100 \%$ throughput for unicast traffic, thus completely solving the drawbacks of single FIFO queue input queueing;

- a packet can be simultaneously sent to multiple destinations, thus efficiently implementing multicasting, by turning on in the same slot more than one wavelength of the transmitter laser array;

- packet transmission is time-slotted and synchronized on all channels (wavelenghts), thus a global packet synchronization is required;

- packets have a fixed length corresponding to one time slot; the packet format adaptation, including segmentation and reassembly is left to higher (electronic) layers of the node protocol.

The proposed architecture tries to combine in an optimal way optical and electronic technologies: the aggregate bandwidth is handled in the photonic domain by working on each wavelength, while packet queueing, MAC protocol and statistical time multiplexing are handled in the electronic domain. At the same time, we tried to focus on a solution that is feasible with optical device, which are available today, and thus use only static optical multiplexer and demultiplexer, EDFA, modulators and an array of fixed wavelength lasers (though we also address the use of fast tunable lasers).

\subsection{RINGO node structure}

The proposed structure for a RINGO node is shown in Fig. 2. The main functions that the node must support are outlined in the following.

- Amplification of the optical signals in order to compensate for the loss of the node passive elements and the downstream fiber link. Amplification is obtained by placing an EDFA at the input of the node. The performance of this EDFA turns out to be of paramount importance for the issue of the node cascadability, and will be analysed in terms of power budget in Sect. 4.1 and gain locking in Sect. 4.2.

- Demultiplexing of the WDM comb after the amplifier. The most promising devices for this purpose are Arrayed Wave-Guide (AWG) filters. 
- Monitoring the wavelengths status on each slot. This is performed by tapping a fraction of the power on each fiber at the output of the demultiplexer and by sending it to a DC-coupled photodiode array. The $\lambda$ monitor, described in Sect. 3, requires an electronics with a much smaller bandwidth than the channel bit-rate. In fact, it should detect the received average power on a packet-by-packet basis, so that its cutoff frequency was chosen to be much lower than the bit rate and of the order of the inverse of the packet duration. This solution seems to be much easier than the approach proposed in [6], where $\lambda$-monitor is achieved using a high frequency subcarrier encoded on each channel, since it requires neither high bandwidth electronic nor encoding at the transmitter side.

- Burst-mode detection of the incoming data-stream on the wavelength $\lambda_{i}$ assigned for the node.

w Packet generation for the local traffic. This is performed on the optical side by a laser array driven by the node controller. The lasers on each wavelength are turned on by injection current direct modulation when a packet has to be generated. The data bits are then "written" inside the packet by an external modulator. Our choice of a laser array for the transmitter has two different motivations:

- to allow efficient multicast. In fact, this transmitter setup exhibits promising features for multicast transmission, since bits are written simultaneously by the external modulator on all wavelengths that are generated by the laser array in a given time slot;

- to allow using commercial and very stable lasers on the ITU grid [2].

An alternative solution is based on a fast tunable laser [11], whose technology is anyway not yet completely available at the commercial level. When available, the tunable laser solution will likely be less expensive than a laser array with a high number of channels.

- Node control. This is performed on a programmable logic device (PLD) that implements the VOQ protocol and several other features, as explained in Sect. 3.1.

\section{Experimental testbed}

The currently implemented testbed is composed of two nodes, each equipped with four wavelengths on a $200 \mathrm{GHz}$ grid: $\lambda_{1}=1550.75 \mathrm{~nm}, \lambda_{2}=1549.16 \mathrm{~nm}$, $\lambda_{3}=1547.52 \mathrm{~nm}$ and $\lambda_{4}=1545.93 \mathrm{~nm}$ as shown in Fig. 3. Two nodes have been implemented, each controlled by its own PLD. The first one acts as a 


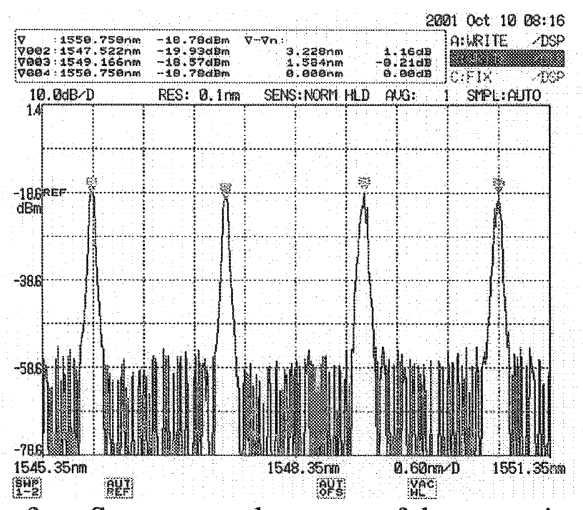

Figure 3. Spectrum at the output of the transmitter node.

traffic generator only, and sends WDM packets to the second one, called "full node" in the following, which implements the full function of the RINGO architecture. Packets are $1 \mu \mathrm{s}$ long, with a $0.1 \mu$ s guardband between each of them, and carry a $2.5 \mathrm{Gbit} / \mathrm{s}$ data traffic, corresponding to approximately 2000 bits per packet. The node is equipped with a DFB laser array, multiplexed into a lithium niobate modulator. Controller packet signals coming from the PLD directly modulate the DFB laser at the chosen wavelength, while the data bits are imposed on the optical packet using the external modulator. Measurements illustrating the MAC protocol and multicast capabilities are shown in Fig. 4. Signals indicating resource occupation, detected by the $\lambda$-monitor device in the full node, are shown in Fig. 4(a): upper trace refer to $\lambda_{1}$ and lower trace to $\lambda_{2}$. Both wavelengths are optically passing through the full node, which

(a)

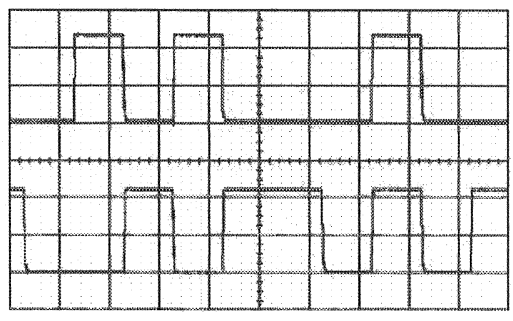

(b)

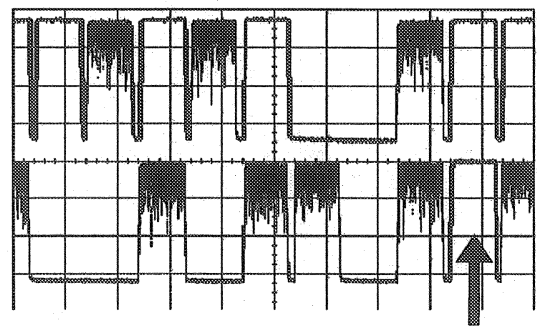

Figure 4. (a) Signal indicating resource occupation for $\lambda_{1}$ (upper trace) and $\lambda_{2}$ (lower trace), taken at the $\lambda$-monitor device output. (b) Traffic at the output of the fully implemented node on $\lambda_{1}$ (upper trace) and $\lambda_{2}$ (lower trace): 'noisy' packets, carrying data bits, are those generated in the node preceding the full node, while 'noiseless' packets are those added by the full node.

adds its local traffic on the empty slots of the same wavelengths. The output traffic on $\lambda_{1}$ (upper trace) and $\lambda_{2}$ (lower trace) is shown in Fig. 4(b). To recognize the origin of the packet, and demonstrate the correct node controller 
operation, we have not written data bits in packets generated by the full node. Therefore, in each trace we can easily determine which packet comes from which node: "noisy" packets (due to the presence of data bits) come from the first node, while "noiseless" packets are inserted by the full node. Looking both at Fig. 4(a) and 4(b), we can observe the implementation of MAC protocol that adds packet only when the resource is available. Also in Fig. 4(b) it is possible to see the multicast capability of the RINGO network: one time-slot (indicated by an arrow) presents a "noiseless" packet on both wavelengths $\lambda_{1}$ and $\lambda_{2}$. This means that the full node is satisfying a multicast traffic request by transmitting the same information on two wavelengths in the same time slot.

\subsection{Hardware implementation of the node logic controller}

The node logic controller was implemented by an electronic board equipped with a high-performance PLD (Altera PROCK20KE600 $100 \mathrm{MHz}$ ). The board can communicate with a workstation via the PCI bus. The function of the PLD is to implement the logic circuits that control the node. It will be programmed by a workstation through a PCI bus.

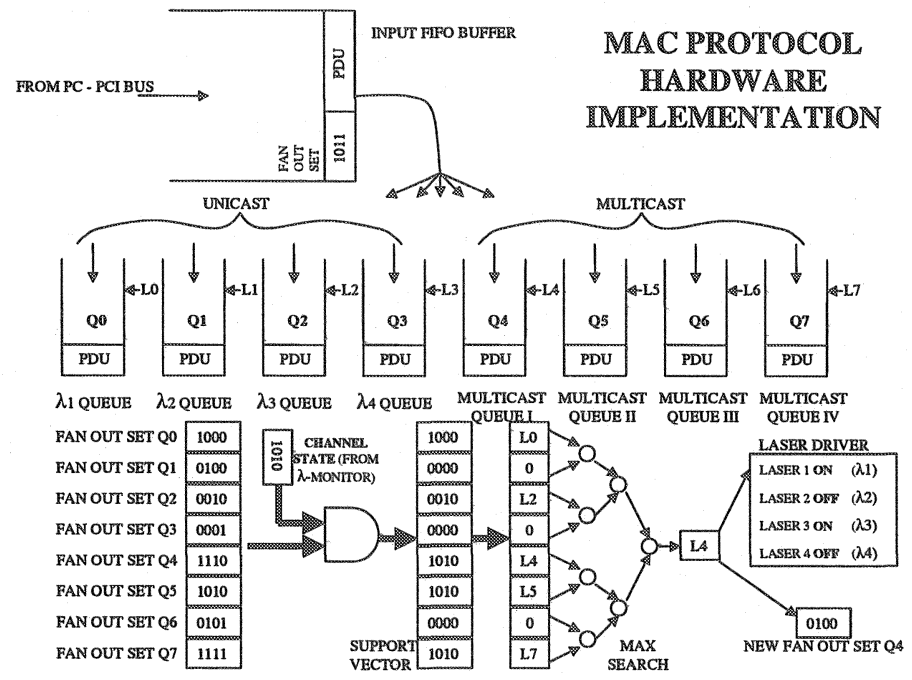

Figure 5. MAC protocol hardware implementation.

The main operations of the MAC protocol hardware implementation is shown in Fig. 5. When a packet arrives from the PCI bus, it is stored into an input FIFO buffer. This buffer is needed to separate the activity of the PCI bus from the activity of the on-board logic. A requirement of the RINGO protocol is to send a waiting packet on the first available slot, if the corresponding wavelength channel is available. Unfortunately, the arrival rate of the packets from the PCI bus is strongly dependent on the workstation operating system, and a storage 
logic on the board is required. Every packet is formatted in a fixed size Protocol Data Unit (PDU) which contains the payload bits (Service Data Unit SDU), and a fan-out set, which codes the information on the packet destination(s). Since four wavelengths are used in this first prototypal architecture and each wavelength identifies a possible destination, the fan-out set is composed of four bits. Eight FIFO queues are used to store these packets: four unicast queues and four multicast queues. In this configuration, it can be easily shown that there are eleven different multicast fan-outs (including the broadcast). A simple criterion based on the minimum Hamming distance [8] is used to choose one of the four destination queues for the multicast packets. In the example of Fig. 5 a transmission request is coded with a logic "1", i.e., the first packet of queue Q6 has to be transmitted on $\lambda_{2}$ and on $\lambda_{4}$. The length of each queue is stored in a special register file (L0.. L L7). On the rising edge of the slot synchronization signal, the channel state is acquired by the $\lambda$-monitor. In the example of Fig. 5 an available wavelength is coded by a logic " ${ }^{61}$ ". In this example, $\lambda_{2}$ and $\lambda_{4}$ are not available. A bitwise AND operation is computed between the channel state vector and the residual fan-out set vector of the head-of-the-line packet of each queue. The result is loaded into a support vector. Some queues cannot be chosen for transmission (in the example Q1, Q3, Q6) because all the wavelengths of their fan-out set are not available. The next step is to find the queue with maximum length among queues having transmission possibility. The maximum length is found by a tournament algorithm. The transmission requests of the "winner" queue, in the example Q4, have to be served in accordance with the channel wavelength availability. It is demonstrated that this choice guarantees the maximum throughput when the queues are not overloaded [8]. In the example, the node controller enables laser 1 and laser 3 to transmit, and it sends the PDU of the first packet in queue 4 to the external laser modulator. The last step is to refresh the queue content. In the example, the fan-out set of the first packet in queue 4 has to be changed from 1110 to 0100 because $\lambda_{2}$ is the only transmission request not served. When all transmission requests are served, the packet is removed by the queue head and the queue fan-out restored.

In the first release of the project, data are not downloaded by the PCI bus, but they are generated on the board by a traffic simulator. This is implemented by a Pseudo-Random Binary Sequence generator controlled by the corresponding Control Unit.

Four different concurrent processes work to control the node. Besides the traffic generation process, the second process is the input buffer manager process, which takes care of receiving the packets from the traffic generator and to store them in the input FIFO buffer. The third process is the queue manager process, which extracts each packet from the input FIFO buffer and moves it to the proper queue. The last process is the core of the system. This process implements the MAC protocol transmitting one or more (in the multicast case) 
packets according to the rules of the protocol. The other processes have to work in transparent mode with respect to this last process. This means that the procressing time from the rising edge of the synchronization signal to the transmission of a packet has to be constant in every traffic situation. Hence the MAC protocol manager process has strict priority on the other processes and, if it needs a shared resource, it can stop the other processes using control signals. For this reason, the other processes are designed to save the current job in dedicated support registers during one clock cycle, in order to give as soon as possible the control of the resources to the MAC protocol manager process.

In future developments of the project, an on-board DSP will directly communicate with the PCI bus. This will require the development of a software driver to get the packets from the RAM of a standard PC or workstation. This software driver will connect the IP and MAC protocol layers with our control units in order to run standard Internet applications.

\section{Optical Transmission Issues}

The RINGO network presents several transmission issues that are common to standard optical point-to-point transmission, such as ASE noise accumulation and power budget control, but also other problems that are typical of packet switched transmission, such as transient effects in the EDFA gain response. The following subsections address some of these issues in detail

\subsection{Power Budget}

The power budget of the RINGO network has been designed by taking into account that:

me thenuation of one span is close to $20 \mathrm{~dB}$. In the following, we indicate as "span" the cascade of the node and the following fiber, as shown in Sect. 3 (15 dB for passive devices in the node and $5 \mathrm{~dB}$ for the fiber link, assuming a $25 \mathrm{~km}$ fiber);

- fiber non-linear effects are negligible, due to the fact that the EDFA is placed at the input of the node, thus its output power is attenuated by $15 \mathrm{~dB}$ before going into the fiber;

- each span should have an input-output gain of $0 \mathrm{~dB}$, i.e., the power budget should be set so that, on the signal wavelengths, the EDFA gain is approximately equal to the span attenuation, thus obtaining a span transparency condition;

- depending on its destination, a packet in RINGO should travel along a number of nodes, whose maximum is equal to the total number of nodes in the network minus one (the one where the packet itself is generated). 
Assuming that the signal transmission is linear and considering ASE accumulation only, it can be shown that the Optical Signal-to-Noise Ratio (OSNR) on a $0.1 \mathrm{~nm}$ bandwidth $(\Delta B)$, for a signal going through $N_{\text {span }}$ cascaded spans, is given by:

$$
O S N R_{d B}=P_{o u t, c h}^{E D F}-\alpha_{\text {span }}-F_{E D F A}-10 \log _{10}\left(N_{\text {span }}\right)-(2 h \nu \Delta B)_{\mathrm{dBm}}
$$

where $P_{o u t, c h}^{E D F}$ is the average power per channel expressed in $\mathrm{dBm}$ at the output of the EDFA, $\alpha_{\text {span }}$ is the span passive device attenuation, and $F_{E D F A}$ is the noise figure of the EDFA. Given $\triangle B=0.1 \mathrm{~nm}$ the last term is equal to $-58 \mathrm{dBm}$.

From this result, we can derive a bound on the maximum number of cascaded spans $N_{\text {span }}^{\max }$ that a packet can go through before its OSNR drops below a certain level. The value of $N_{\text {span }}^{\max }$ increases when increasing $P_{\text {out,ch }}^{E D F A}$ and decreasing $\alpha_{\text {span }}$. Thus, a $3 \mathrm{~dB}$ increase in $P_{\text {out }, c h}^{E D F A}$ leads to a potential increase by a factor of two in $N_{\text {span }}^{\max }$.

As an example, if the minimum required $\mathrm{OSNR}_{d B}$ is set to $20 \mathrm{~dB}$ (corresponding approximately to a Q-factor close to $20 \mathrm{~dB}$ at $10 \mathrm{Gbit} / \mathrm{s}$ for a standard OC-192 receiver), in order to have $N_{s p a n}^{\text {max }}=16$, the minimum value for $P_{o u t, c h}^{E D A}$ is $-1 \mathrm{dBm}$. Considering $2 \mathrm{~dB}$ as a system margin, the power per channel at the output of the EDFA should be of the order of $+1 \mathrm{dBm}$. For a standard WDM transmission on 16 channels, this would correspond to a total output power of the order of $13 \mathrm{dBm}$, a value easily achievable by commercial EDFAs. However, due to the burstyness of the packetized optical signals entering the EDFA, gain-locking techniques are required. The techniques proposed so far [13] allow to efficiently reduce the gain transient at the expense of a reduction of the useful output power. It is thus fundamental to optimize the gain-locking stategy in order to obtain, for a given EDFA, the maximum $P_{o u t, c h}^{E D A}$ that gives acceptable gain transient effects under real traffic. This is the focus of the following section.

The other approach to increase $N_{s p a n}^{\max }$ is the reduction of $\alpha_{\text {span }}$. Although 1 or $2 \mathrm{~dB}$ attenuation reduction can be envisioned for our node structure by trying to optimize passive components

\subsection{Gain Locking Experiment}

It has been shown [13] that systems where the optical signal carries burstmode packet traffic and uses EDFAs for amplification must cope with timedependent output power and OSNR fluctuations due to transient effects in the EDFA gain. Gain fluctuation effects become particularly relevant when packet signals travel along a quite long chain of EDFAs [14]. The proposed countermeasure is some form of gain locking of the EDFAs by injecting a proper out-of-band "locking" signal at the input of the amplifier. This technique has been experimentally studied within the RINGO project. The target of our ex- 
(a)

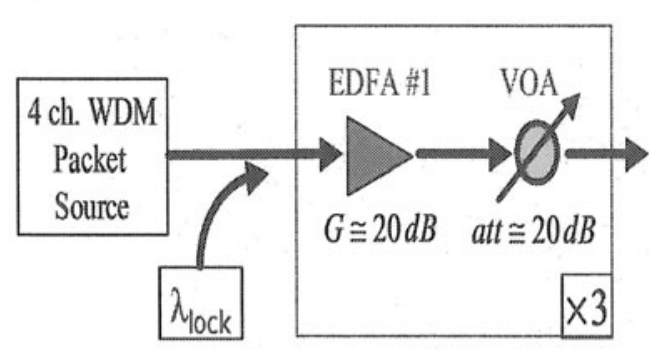

(b)

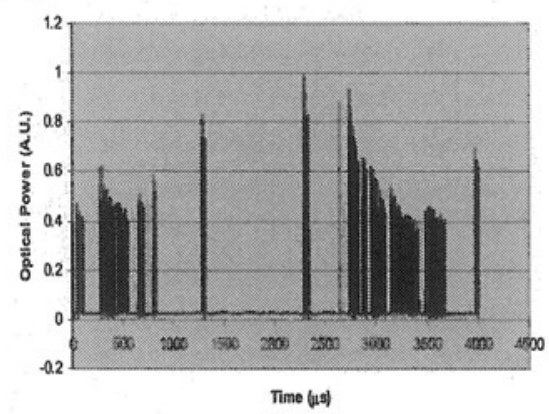

Figure 6. (a) System experimental setup. (b) Measured time-dependent power fluctuation at the output of the EDFA, without gain locking, single channel, $50 \%$ load $T_{\text {on }}+T_{\text {off }}=400$ slots, $P_{c h}=-30 \mathrm{dBm}$.

periment is finding a gain locking configuration that, for the available amplifiers (DITECH STAR L-16, $16 \mathrm{dBm}$ nominal saturation power), determines what is the maximum EDFA input power that gives an acceptable gain fluctuation on the output signal under realistic burst-traffic conditions at the input. We investigated on the actual system impact of this effect. This has been carried out as follows.

- We introduced a new parameter $Q_{\Delta G}$ that can experimentally quantify the gain fluctuations in a given system. The parameter has been chosen in order to be easily measurable on the received eye diagram, and it is defined as:

$$
Q_{\Delta G}=\frac{E}{\sigma_{\Delta G}}=\frac{m_{1}-m_{0}}{\left(\sigma_{\text {tot }}^{1}-\sigma_{\text {noise }}^{1}\right)-\left(\sigma_{\text {tot }}^{0}-\sigma_{\text {noise }}^{0}\right)}
$$

where $E=m_{1}-m_{0}$ is the average eye opening, $m_{1} /\left(m_{0}\right)$ being the average levels for a received " $1 " /\left(\right.$ " 0 "), and $\sigma_{\Delta G}$ is the standard deviation of eye levels due to the gain fluctuation effects only. $\sigma_{\Delta G}$ can be estimated as $\left(\sigma_{\text {tot }}^{1}-\sigma_{\text {noise }}^{1}\right)-\left(\sigma_{\text {tot }}^{0}-\sigma_{\text {noise }}^{0}\right)$, where $\sigma_{\text {tot }}^{1} /\left(\sigma_{\text {tot }}^{0}\right)$ is the standard deviation for a received " $1 " /\left({ }^{\prime \prime} 0\right.$ ") under bursty traffic, while $\sigma_{\text {noise }}^{0},\left(\sigma_{\text {noise }}^{0}\right)$ is the standard deviation when the gain fluctuation has been removed. These two quantities can be easily measured by letting the system run without packetization (i.e., with signal ON in every slot). It is worth noting that $Q_{\Delta G}$ tends to low values when gain fluctuations are relevant, while ideally, it should be as high as possible.

- We correlate this parameter with actual penalty on BER measurements, in order to estimate the expected system penalty for a given $Q_{\Delta G}$ for both gain-locked and unlocked situations. 
We used the experimental setup shown in Fig. 6(a). The signal source was a bank of four WDM lasers on the ITU grid $(1545.93 \mathrm{~nm}, 1547.52 \mathrm{~nm}, 1549.16 \mathrm{~nm}$ and $1550.75 \mathrm{~nm}$ ). Each laser was directly turned on-off by four parallel pattern generators that have been programmed to emulate independent packet sources with slot time equal to $1 \mu \mathrm{s}$. The source traffics have been modeled according to independent $\mathrm{ON} / \mathrm{OFF}$ cell arrival processes based on geometrically distributed random number generators [13]. The free parameters of the arrival processes are $T_{o n} /\left(T_{\text {off }}\right)$, equal to the average number of consecutive $\mathrm{ON}(\mathrm{OFF})$ slots. The average traffic load is thus given by $T_{o n} /\left(T_{o n}+T_{o f f}\right)$, while the $T_{o n}+T_{o f f}$, gives an indication of the traffic burstiness. The four wavelengths were sent to an external modulator, driven with a $2^{20}-1$ pseudorandom bit stream at $2.5 \mathrm{Gbit} / \mathrm{s}$. The average power per channel at the output of the transmitter was $P_{c h}$, measured when setting the packet generation to "ON" in all slots. A $\mathrm{CW}$ laser source at wavelength $\lambda_{\text {lock }}$ and power $P_{\text {lock }}$ was added in order to gain-lock the following amplifiers. The resulting optical signal was sent to a link with three identical sections, each made by an EDFA followed by a $20 \mathrm{~dB}$ optical attenuator, emulating the loss between two nodes of an optical network. The gain of each EDFA was set to compensate for the attenuator loss. Sets of measurements were taken changing several system parameters, such as the number of WDM channels, the characteristic of the packet traffic, the channel power $P_{c h}$. Fig. 6(b) shows the typical output of the system when $P_{l o c k}=0$ (no locking) for a single channel system, load $50 \%, T_{\text {on }}+T_{\text {off }}=400$ slots, $P_{c h}=-30 \mathrm{dBm}$. The oscilloscope time-base was set in the $\mu$ s scale in order to follow the packet envelope, and thus the gain fluctuation, while the $2.5 \mathrm{Gbit} / \mathrm{s}$ bits are not visible. Significant fluctuations with time scale of the order of hundreds of $\mu$ s are evident.
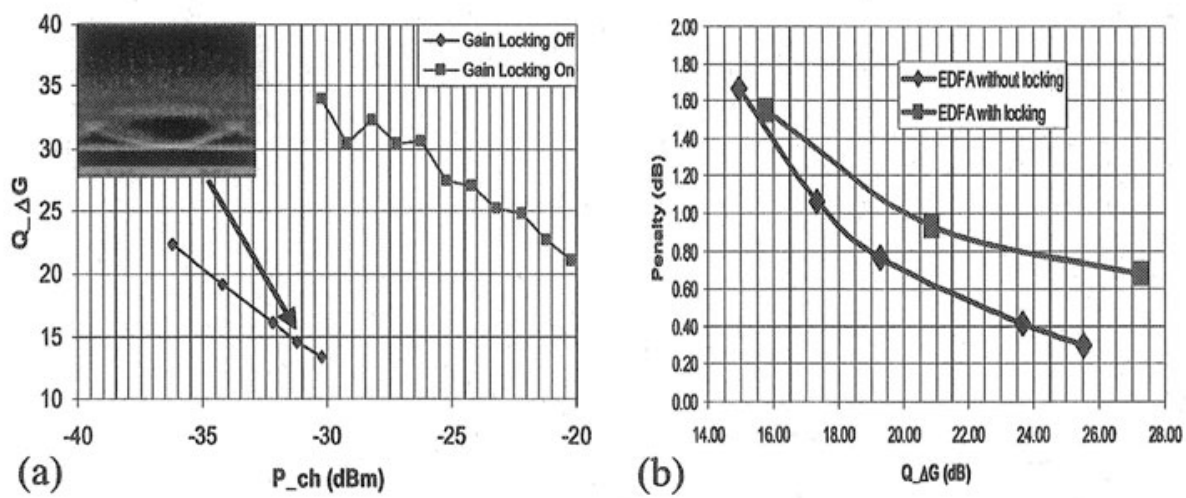

Figure 7. (a) $Q_{\Delta G}$ vs. $P_{c h}$ for a single channel system, with and without gain locking. The upper left corner of the graph reports the eye diagram at $-30 \mathrm{dBm}$. (b) Sensitivity penalty at $10^{-9}$ vs. $Q_{\Delta G}$, with and without gain locking. 
Fig. 7(a) shows $Q_{\Delta G}$ vs. $P_{c h}$ in the same situation, both with no locking $\left(P_{l o c k}=0\right)$ and with a locking power turned on $\left(P_{l o c k}=-16 \mathrm{dBm}\right) \cdot Q_{\Delta G}$ increases when decreasing $P_{c h}$. The beneficial effects of locking are evident since the same value of $Q_{\Delta G}$ can be achieved for approximately $15 \mathrm{~dB}$ higher $P_{c h}$ than in the locked case. A typical eye diagram for $Q_{\Delta G}=14 \mathrm{~dB}$ is shown as an example in the upper-left corner of the figure. In order to correlate $Q_{\Delta G}$ with system penalty, we measured the BER of the system, estimating receiver sensitivity penalty in different situations. Fig. 7(b) shows the penalty vs. $Q_{\Delta G}$ when locking is turned on or off. The two plots differ due to different gain fluctuation statistic, but it is important to note that the $1-\mathrm{dB}$ sensitivity penalty at $\mathrm{BER}=10^{-9}$ is achieved when $Q_{\Delta G}$ is in the range $18-20 \mathrm{~dB}$, and that the penalty is negligible for $Q_{\Delta G}>22 \mathrm{~dB}$. We found a similar penalty- $Q_{\Delta G}$ relation for other system setups, indicating that the parameter $Q_{\Delta G}$ can be a useful way to quantify the system impact of gain fluctuations. A useful rule of thumb in the design of a system is the fact that $Q_{\Delta G}$ should be greater than 18-20 dB to have a sufficiently low system impact. We have then measured $Q_{\Delta G}$ vs. $P_{c h}$ for a four-channel system, with gain locking turned on, in order to study the impact of different traffic models. Fig. 8(a) shows the results
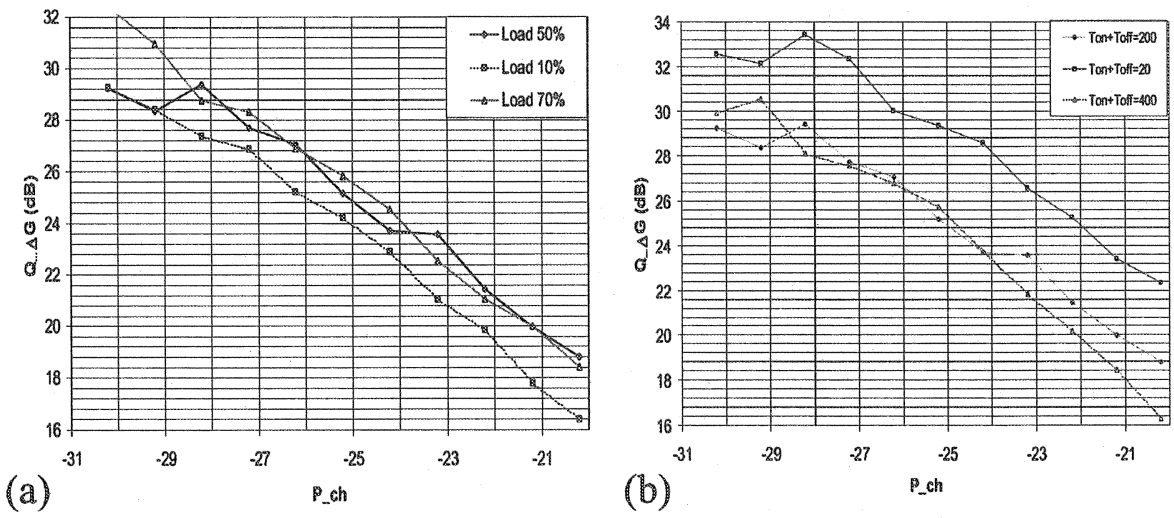

Figure 8. Four channel experiment: (a) $Q_{\Delta G}$ vs. $P_{c h}(\mathrm{dBm})$. Curves for $T_{o n}+T_{o f f}=$ 200 slots and variable loads $(10 \%, 50 \%$ and $70 \%)$. (b) $Q_{\Delta G}$ vs. $P_{c h}(\mathrm{dBm})$. Curves for load $=50 \%$ and variable $T_{o n}+T_{o f f}=20,200$ and 400 slots.

when $T_{o n}+T_{o f f}=200$ slots, with different traffic loads. We notice that the performances are worst for low loads $(10 \%)$, where $Q_{\Delta G}=20 \mathrm{~dB}$ is reached for approximately $1 \mathrm{~dB}$ less in $P_{c h}$ than in the $50 \%$ and $70 \%$ loads, which in turn both give a similar behavior. This result agrees with the theoretical predictions of [13], being due to the fact that for lower traffic the probability of isolated packets after a long sequence of idle slot is higher. During long idle sequences, the population inversion in the EDFA grows, giving rise to higher available 
gains for the following packets. We also investigated the effect of changing the traffic burstiness by varying $T_{o n}+T_{\text {off }}$ while leaving the load fixed at $50 \%$. The higher is $T_{o n}+T_{o f f}$, the higher is the probability of long sequence of consecutive ON slots. The results are shown in Fig. 8(b). We remark that the gain fluctuation is lower for smaller $T_{o n}+T_{o f f}$, since it corresponds to more uniform traffic. In all the considered situations, even with gain locking turned on, the power per channel $P_{c h}$ should always be kept below approximately -22 $\mathrm{dBm}$, corresponding to a total useful EDFA output power of $+4 \mathrm{dBm}$, a much lower level than the EDFA nominal output saturation power $(16 \mathrm{dBm})$. Efficient gain locking is thus a fundamental issue in any true packet optical network.

\section{Discussion and Conclusion}

This work has been motivated by the idea that optical packet transmission, though neither yet standardized nor commercially available, may soon become a promising alternative to the current approach, based on building WDM networks with a high degree of fast "circuit-switching" reconfigurability, but where packet switching is still completely implemented at the electronic level. This is the major innovation for the RINGO project presented in this paper. We have shown in detail the fundamental features of the architecture and implemented MAC protocols, which are suitable for a high capacity WDM ring of MAN/WAN extension (target of 16 nodes with up to $25 \mathrm{~km}$ between nodes).

Several network subsystems have been experimentally demonstrated and tested, showing that the proposed architecture is feasible with today's commercial components, without necessarily requiring components such as fast tunable lasers, fast tunable filters/switches and wavelength converters that, though extensively studied in R\&D labs, are not yet stable enough to become commercially available devices.

Furthermore, we will study, both experimentally and by simulation, the feasibility of a alternative node structures, by considering the use of fiber Bragg grating as alternatives to AWG-based filters.

\section{Acknowledgments}

The work was funded by MURST (Italian Scientific Research and University Ministry), project COFIN 99. The authors wish to thank the companies that have donated some of the components used in our testbed: Lucent Technologies Italia for the TrueWave RS fibers, ITALTEL for the AWG filters, and CISCO Photonics for Drivers and Modulators.

\section{References}

[1] R. Ramaswami and K. N. Sivarajan, Optical Networks - A Practical Perspective, Morgan Kaufman, 1988.

[2] ITU-T Recommendation G.872 
[3] R. E. Wagner et al, "MONET: Multiwavelength Optical Networking," IEEE/OSA Journal of Lightwave Technology, vol. 14, pp. 1349-1355, June 1996.

[4] S. Yao et al, "Advances in Photonic Packet Switching: an Overview", IEEE Communication Magazine, Vol. 38, No. 2, pp. 84-94, Feb. 2000.

[5] A. Carena et al, "OPERA: an optical packet experimental routing architecture with label swapping capabilities", IEEE/OSA Journal of Lightwave Technology, Vol. 16, No. 12, pp. 2135-2145, Dic. 1998.

[6] K. V. Shrikhande et al, "HORNET: a packet-over-WDM multiple access metropolitan area ring network" IEEE Journal of Selected Areas in Communications, Vol. 18, No. 10, pp. $2004-2016$, Oct. 2000.

[7] C. Guillemot et al, "Transparent optical packet switching: the European ACTS KEOPS project approach", IEEE Journal of Lightwave Technology, Vol. 16, No. 12, pp. 2117-2134, Dec. 1998.

[8] N. McKeown et al, "Achieving 100\% throughput in an input-queued switch", IEEE Transactions on Communications, Vol. 47, No. 8, pp. 1260-1267, Aug. 1999.

[9] A. Tajuma, "A 10-Gbit/s optical asynchronous cell/packet receiver with a fast bit synchronization circuit, OFC'99 Tech. Dig., San Diego, CA, Tul6, pp. 111-113

[10] M. Ajmone Marsan et al, "MAC Protocols and Fairness Control in WDMMulti-Rings with Tunable Transmitters and Fixed Receivers," IEEE/OSA Journal on Lightwave Technology, Vol. 14, No. 6, pp. 1230-1244, June 1996.

[11] P. J .Rigole et al, "Fast wavelength switching in a widely tunable GCSR laser using a pulse pre-distortion technique", OFC'97 Tech. Dig., Dallas, TX, WL63, pp. 231-232.

[12] M. Shell et al, "Improved crosstalk performance in a 10-wavelength 25 Gbps multichannel laser array transmitter module", 1997 IEEE/LEOS Summer Topical Meeting Tech. Dig., 1997, pp. 64-65.

[13] M. Karasek et al, "Gain Stabilization in Gain Clamped EDFA Cascades Fed by WDM Burst-Mode Packet Traffic", IEEE/OSA Journal on Lightwave Technology, Vol.18, No.3, pp. 308-313, Mar 2000.

[14] A. Mecozzi et al, "Theory of optical amplifier chains", IEEE/OSA Journal of Lightwave Technology, Vol. 16, No. 5, pp. 745-756, May 1998.

[15] M. Schwartz, Broadband Integrated Networks, pp. 45-58, Prentice Hall, 1996.

[16] http://www.artis-software.com/products/optsim 